\title{
Acute Myeloid Leukemia: A New Era of Therapies, a New Wave of Toxicities?
}

\author{
Andrew M. Brunner \\ Massachusetts General Hospital, Harvard Medical School, Boston, MA, USA
}

Few malignancies have seen such a dramatic tidal change in treatment decision-making as has acute myeloid leukemia (AML). Between 2017 and 2018, a total of eight new therapeutic agents were approved by the United States Food and Drug Administration (FDA) for the treatment of AML, either for the initial treatment of the disease, or for AML that is relapsed or refractory. These agents include therapies targeting specific mutations in AML, including midostaurin or gilteritinib that target mutated FMS-like tyrosine kinase-3 (FLT3), enasidenib that targets mutated isocitrate dehydrogenase (IDH)-2, and ivosidenib that targets mutated IDH1 proteins. There have also been approvals for therapies that may have an effect on broader groups of AML patients not dependent on the presence of a specific mutation, including (a) gemtuzumab ozogamicin that is directed toward CD33, CPX351; or (b) vyxeos, which is a liposomal formulation of cytarabine and daunorubicin; (c) glasdegib that is an inhibitor of the sonic hedgehog pathway; and (d) venetoclax that effects apoptosis by targeting BCL2.

Together, these newly approved agents provide a new arsenal for treating AML, both as single agents and with the prospect of new therapeutic combinations in the future. Rapidly, the treatment of AML has been made more effective in many ways, but also far more complicated. Moreover, while the number of patients treated on seminal trials has been encouraging for an uncommon malig-

\section{KARGER}

(c) 2019 S. Karger AG, Basel

E-Mail karger@karger.com

www.karger.com/aha

Editorial comment on the paper by Shai Shimony
et al. Necrotizing Hemorrhagic Gastritis following
Acute Myeloid Leukemia Induction with Midostaurin:
An Unexpected Complication. A Case Report. Acta
Haematol 2020;143:65-68

nancy, the absolute number of patients treated with these agents is still relatively small. The largest of these studies, the RATIFY trial, randomized 717 patients with FLT3mutated AML to receive standard induction and consolidation or chemotherapy combined with the FLT3-inhibitor midostaurin [1]. A total of 360 patients were treated on the arm containing midostaurin; the reported toxicity profile was overall similar between those receiving midostaurin and those given placebo, with some signals for slower count recovery in the midostaurin arm. Interestingly, the known toxicity profile of midostaurin monotherapy, particularly gastrointestinal toxicity, was not clearly seen in combination with chemotherapy, possibly because of the high baseline toxicity of induction chemotherapy.

This illustrates a challenge, as more patients start to be treated with these agents in routine clinical practice. The reality is that we may yet encounter relatively uncommon but significant toxicities that should inform the way that the leukemia community approaches them. A good example may be seen in the case of inhibitors of mutated IDH1 and IDH 2 proteins in AML. Approximately $10 \%$ of the patients treated with ivosidenib or enasidenib, respectively, may develop a phenomenon characterized as "differentiation syndrome" associated with leukocytosis and maturing peripheral blood cells as well as dyspnea, fever, and weight gain, and which can be life threatening if not 
recognized early [2]. Fortunately, it can generally be treated with steroids and other supportive care, usually not requiring treatment cessation. Identifying emerging risks associated with the growing number of available therapies in AML - and in other cancers as well - is critical to both counsel patients and ideally to develop strategies to mitigate or prevent severe toxicity.

In this issue of Acta Haematologica, Shimony et al. [3] describe their experience in treating a patient with a new AML diagnosis with standard $7+3$ induction combined with midostaurin, based on the dosing used in the RATIFY trial [1]. They describe a patient who developed necrotizing hemorrhagic gastritis that appeared to be related to midostaurin given the timing of the event and exposure to other drugs. In this case, there was slow resolution over time and with total parenteral nutrition and proton pump inhibitor therapy; the patient received further standard chemotherapy but did not have re-exposure to midostaurin. Gastritis in general was relatively rare on the RATIFY trial - only $3 \%$ of the patients - and no cases of necrotizing hemorrhagic gastritis were reported.

In this case, the clinical team recognized the potential role of midostaurin early and ceased further treatment based on identifying this rare event. Many newly ap- proved agents are now available in the treatment of AML, but these approvals were based on, overall, a small number of patients enrolled to a given study. As such, these "rare events" are likely to become more common. Indeed, it is important that follow-up and post-marketing studies with larger numbers of patients monitor for toxicity signals that may not have been reported to date. Not only will these agents likely be combined with other treatments, making it potentially more difficult to identify unique toxicities, it is also likely that they may be used outside of their intended label, as has been seen with other therapies that have narrow indications as a part of their approval [4]. The arrival of these new therapies in AML has greatly improved the outlook for so many clinicians and patients; it is just as important, in these early days, to identify the potential toxicities - even rare ones - that they may bring.

\section{Disclosure Statement}

Dr. Brunner has served on advisory boards for Celgene and Jazz Pharmaceuticals, received honorarium from Xcenda, and reports clinical trial research funding to his institution from Novartis, Celgene, Takeda, H3Biomedicine, and AstraZeneca.

\section{References}

1 Stone RM, Mandrekar SJ, Sanford BL, Laumann K, Geyer S, Bloomfield CD, et al. Midostaurin plus Chemotherapy for Acute Myeloid Leukemia with a FLT3 Mutation. N Engl J Med. 2017 Aug;377(5):454-64.

2 Fathi AT, DiNardo CD, Kline I, Kenvin L, Gupta I, Attar EC, et al.; AG221-C-001 Study Investigators. Differentiation Syndrome Associated With Enasidenib, a Selective Inhibitor of Mutant Isocitrate Dehydrogenase 2: Analysis of a Phase 1/2 Study. JAMA Oncol. 2018 Aug;4(8):1106-10.
3 Shimony S, Mintz HRR, Beryozkin YS, Shoham A, Raanani P, Wolach O. Necrotizing hemorrhagic gastritis following AML induction with midostaurin: an unexpected complication. Acta Haematol. DOI: 10.1159/ 000500975 .

4 Brunner AM, Weng S, Cronin A, Fathi AT, Habib AR, Stone R, et al. Impact of lenalidomide use among non-transfusion dependent patients with myelodysplastic syndromes. Am J Hematol. 2018 Sep;93(9):1119-26. 